\title{
Editorial
}

\section{Sintonías de aprendizaje: Sikolo.}

\section{Learning tunes: Sikolo-school.}

Julio C. de la Torre-Montero, Ph.D.

EUEF San Juan de Dios. Universidad Pontificia Comillas.

Editor. Director de la revista SEEO.

juliodelatorre@comillas.edu

SEEO no se hace responsable de las opiniones

vertidas por los autores de los artículos que publica siendo estos de carácter personal.

Mi primera visión de la enfermedad oncológica tuvo lugar a los 12 años, con mi abuelo materno, al que estaba muy unido: se le diagnosticó un cáncer de pulmón; después de unos años se repetiría la noticia con mi abuelo paterno. Todo esto, antes de comenzar la época universitaria, pero que posiblemente marcó un punto de inflexión en las decisiones académicas, vitales y profesionales posteriores.

Cada uno de nosotros, trabajando en oncología, tenemos mucho que decir: biografías particulares de múltiples aristas, luces, sombras y muchas experiencias a pie de cama, de sillón de hospital de día y de silla de sala de espera. Tenemos una historia que contar.

En mis primeras etapas, hace dos décadas, en un hospital de día oncológico las cosas eran sencillas en lo técnico: pocas vías centrales (aunque las periféricas requerían destreza con las palomillas metálicas, ya que aún no se utilizaba el Abbocath ${ }^{\text {TM }}$ de forma habitual) y esquemas de tratamiento sencillos (esquema Mayo para colon, CHOP para Linfomas, CMF para Mama, seguido de Antraciclinas y sus diferentes fórmulas liposomales, Cisplatino para Pulmón, etc.). En todo esto, el contacto con el paciente y sus familia, era maravilloso y ciertamente, para cualquier joven profesional, ilusionante e instructivo. Poco a poco y de forma progresiva, fueron sumándose nuevos fármacos, tanto de quimioterapia como de anticuerpos monoclonales, moléculas orales y combinaciones de todos ellos, con nuevos esquemas que podríamos denominar "de esperanza".

Estas circunstancias nos han llevado a disponer de una escuela donde el conjunto de profesionales de la salud implicados en el cuidado del paciente oncológico y su familia, evolucionamos en el aprendizaje. Dicho proceso, ha ido seguido necesariamente de un desarrollo técnico y una revolución farmacológica que llega hasta nuestros días, y donde podríamos afirmar, estamos al comienzo de una nueva era, que requiere seguir trabajando en la línea de la inversión en investigación, por encima de intereses economicistas. 
La vida de ningún paciente, en nuestro país o en cualquier parte del mundo, se puede monetizar de manera simplista, esté en el estado en el que esté y los gobiernos deben ser garantes de la seguridad de los pacientes, ofreciendo la mejor cobertura posible en tratamientos, ya sean farmacológicos, de soporte o de cuidados paliativos cuando llegue el momento, respetando los principios bioéticos más elementales y dejando patente la oferta del mejor tratamiento posible para cada situación, hacia los límites de lo mejor que podamos dar: towards the best through the better: from within the deepest of our knowledge.

Sikolo significa colegio o escuela, en el idioma Bulu, del África ecuatorial central, y en muchos idiomas africanos bantúes, con pequeñas diferencias. En muchos países, la significación de la escuela representa, no sólo el lugar de instrucción, sino la salida hacia un mundo dónde adquirir conocimientos es considerado como un privilegio, y una oportunidad de ofrecer un futuro mejor a sus comunidades.

Para muchos de nosotros, siguiendo el paralelismo de la educación, el hospital complementa la formación recibida. En ese sentido, no podremos utilizar el verbo completar, sino complementar, pues cuanto más tiempo llevamos en ello, más conscientes somos de lo que nos queda por aprender, una necesidad vital que después se verá reflejada en el crecimiento profesional basado en la experiencia.

Los idiomas como vehículo de comunicación $^{1}$ permiten que el conocimiento sea compartido. Esto se puede aplicar a los diferentes niveles de comprensión lingüística, con una terminología en inglés que se denomina
Codeswitching. Cómo hacer del lenguaje un sistema donde la adaptación a los registros de nuestros interlocutores (nuestros pacientes) sea efectivo: en un escenario en el ámbito de la oncología en el que los conceptos sobre bioquímica, metabolismo celular y comportamiento inmunológico son claves, tendremos que hacer un esfuerzo es la acomodación lingüística de la terminología oncológica de comunicación con el paciente.

En oncología como ciencia, vamos mucho más allá en la necesidad de aprendizaje. Los conocimientos se renuevan constantemente. La comprensión cada vez más profunda de la oncogénesis, seguida de la puesta en marcha de ensayos clínicos permiten el desarrollo final de la investigación básica. El estudio de nuevas vías de bloqueo metabólico en el desarrollo tumoral, con diferentes trabajos que relacionan los procesos bioquímicos celulares con la posibilidad de cercar el crecimiento tumoral y acabar con el mismo son una constante en las nuevas publicaciones científicas.

Los últimos datos sobre prevalencia del cáncer ${ }^{2}$ hablan de un aumento, tanto de esa prevalencia, como de la incidencia en los próximos años. Debemos el aumento de la prevalencia, afortunadamente, a las mejoras continuas en los tratamientos, y a cotas de supervivencia que van en aumento cada año. El cáncer de mama es un ejemplo de ello, así como el cáncer de pulmón y melanoma, dos grandes bienvenidos a estas cifras que hablan de remisiones completas con unas medianas de supervivencia nunca vistas hasta ahora.

La SEEO ya trabaja en varios frentes: diferentes grupos de trabajo: fichas de quimioterapia, que añaden nuevos 
fármacos, supervivencia, trasplantes hematopoyéticos, pediatría, vascular, etc. En muchos de estos casos, el modo de trabajo es multidisciplinar, lo cual enriquece y mejora la atención al paciente.

Para atender estas necesidades, además de las que clásicamente atendemos, hemos de capacitar y entrenar a nuevos profesionales, que con la misma ilusión, o mayor, nos den el relevo en lo asistencial, docente e investigador, además de la gestión, como facilitador de lo anterior.

Todo esto nos lleva a que, como en tantas ocasiones, afirmemos que la especialización profesional en enfermería oncológica es de facto una realidad. Solamente queda la voluntad política a la que llamamos con la responsabilidad de poner en marcha los mecanismos necesarios para que pueda desarrollarse plenamente. Nuestros compañeros, y especialmente los pacientes, lo agradecerán.

Este número en digital de la revista SEEO es un claro ejemplo de la evolución natural de nuestra especialidad: revisión ciega por pares, plataforma digital de admisión de artículos y una indexación cada vez más amplia, y, sobre todo, permite el acceso abierto y gratuito a la literatura científica en español, para cualquiera que quiera leer y aprender de sus contenidos, además de publicar y contribuir con los propios conocimientos y resultados de sus investigaciones.
Recogemos en este primer número la locución: Stay hungry, stay foolish, palabras de Steward Brand ${ }^{3}$, despidiendo a su vez el último número de la revista Whole Earth Catalog, y que Steve Jobs mencionaría en el discurso de Graduación de los alumnos en la Universidad de Standford ${ }^{4}$ en el año 2005: "permaneced hambrientos, mantened la locura..." de conocimiento, de innovación, y de ideas que puedan cambiar el mundo.

De nuestros pequeños mundos, también.

\section{Referencias:}

1. Saohatse MC. African language varieties at Baragwanath Hospital: a sociolinguistic analysis. Thesis. (1997). University of South Africa.

2. Arnold, Melina et al. Progress in cancer survival, mortality, and incidence in seven highincome countries 1995-2014 (ICBP SURVMARK-2): a population-based study. The Lancet Oncology, (2019) Volume 20, Issue 11, 1493 - 1505

3. Cadwalladr, C. Stewart Brand's Whole Earth Catalog, the book that changed the world. (2013). The Guardian. Accessed Nov 3, 2019.

4. University Stanford. Text of Steve Jobs' Commencement address (2005). 2005; Available at: https://news.stanford.edu/2005/06/14/jobs061505/. Accessed Nov 3, 2019. 